Des fictions «toutes proches »: une certaine identité de la France. Enjeux politiques des séries télévisées de France 3 en prime time (Louis la Brocante, Famille d'accueil, Un village français)

\title{
Benoit Lafon
}

\section{OpenEdition}

\section{Journals}

Édition électronique

URL : https://journals.openedition.org/mots/20694

DOI : $10.4000 /$ mots.20694

ISSN : 1960-6001

\section{Éditeur}

ENS Éditions

\section{Édition imprimée}

Date de publication : 15 septembre 2012

Pagination : 79-95

ISBN : 9782847883732

ISSN : 0243-6450

\section{Référence électronique}

Benoit Lafon, «Des fictions « toutes proches » : une certaine identité de la France. Enjeux politiques des séries télévisées de France 3 en prime time (Louis la Brocante, Famille d'accueil, Un village français) », Mots. Les langages du politique [En ligne], 99 | 2012, mis en ligne le 15 septembre 2014, consulté le 23 avril 2022. URL : http://journals.openedition.org/mots/20694; DOI : https://doi.org/10.4000/mots. 20694 


\section{Des fictions " toutes proches " : une certaine identité de la France. Enjeux politiques des séries télévisées de France 3 en prime time (Louis la Brocante, Famille d'accueil, Un village français)}

La fiction, et notamment la fiction télévisée de par l'implication qu'elle propose à ses publics, constitue un fait social essentiel à comprendre. "Feintises ludiques» (Schaeffer, 1999; Odin, 2000), elles proposent aux téléspectateurs de «faire semblant» le temps d'une histoire, d'oublier l'espace environnant pour être «mis en phase », immergés. Proposer de tels récits est le travail quotidien d' «artisans de l'imaginaire » (Dagnaud, 2006), producteurs, scénaristes et diffuseurs.

Nous nous intéressons ici à une configuration spécifique de cet «artisanat» : celle des fictions diffusées en prime time (après 20 h30) sur France 3, présentées sur le site de la chaîne comme «toutes proches». Notre point de départ se fonde sur la prise en compte des meilleurs scores d'audience de la chaîne chaque année depuis 2005 (statistiques de Médiamétrie, avec les limites que cela suppose : voir Macé, 2006, p.76 et suiv.). Il s'agit de saisir à la fois comment France 3 assoit son identité discursive de télévision régionale et comment, ce faisant, cette chaîne contribue à construire un « espace identitaire collectif» national (Dagnaud, 2006, p. 244). Nous avons constitué notre corpus en retenant les quelques fictions ayant chaque année dépassé le score de 5 millions de téléspectateurs en prime time. Il s'est avéré que trois séries seulement dominaient nettement les audiences sur les sept ans de la période 2005-2011 (hormis Plus belle la vie, seule série diffusée avant 20 h 30) : Louis la Brocante, Famille d'accueil, Un village français. En fin de compte, notre corpus est constitué des dix épisodes à forte audience issus de trois séries : six épisodes de Louis la Brocante, deux épisodes de Famille d'accueil et deux épisodes de Un village français (que nous nommerons dans la suite de l'article LLB, FA et VF; le détail des épisodes est consultable infra, tableau 1). 
Nous développons notre analyse en deux points. En premier lieu, il s'agit de caractériser la «politique de fiction » de la chaîne France 3 afin de comprendre comment le modèle français de télévision régionale cherche à représenter le territoire (Esquenazi, 1997; Lafon, 2000), et ce de manière variée à l'aide de formules renouvelées. Dans un second temps, une démarche fondée sur une «sociologie pragmatique des personnages» en rapport avec la «sociologie des processus de création » (Chalvon-Demersay, 2005, p. 79-80) sera menée, tout d'abord par l'analyse des «mises en phase» des téléspectateurs (Odin, 2000), puis par l'analyse des personnages, véritables incarnations identitaires françaises; nous terminerons par la recherche du leitmotiv de chaque épisode, permettant de mieux saisir les ressorts politiques sous-jacents à ces séries.

\section{France 3, la fiction et les séries : entre héritage d'un service public et mutation des formules produites}

«Le territoire d'activité d'un média est d'abord national, et éventuellement régional ou local. On oublie combien les médias œuvrent sur une matrice identitaire nationale, [...] combien ils reflètent, par un prisme singulier, une histoire et une culture circonscrites à un territoire» (Dagnaud, 2006, p. 37). National, le travail des médias se construit en adéquation avec les missions qui leur sont confiées. Principale héritière du monopole public sur l'audiovisuel, France 3, la chaîne nationale publique des régions, s'intègre plus que toute autre dans un cadre spécifiquement national, d'autant plus qu'en l'absence de prime time régionalisé, elle diffuse un programme national et en particulier des fictions devant figurer le caractère régionalisé de l'antenne, dans un contexte de production aujourd'hui largement externalisé. Comment parvient-elle à articuler ces dimensions régionale et nationale? Quelles fictions sont-elles notables dans ce contexte? À quels publics ces fictions s'adressentelles, et quel « espace identitaire collectif» (Dagnaud, 2006, p. 244) - ou quels espaces - proposent-elles aux téléspectateurs?

La politique de fiction de la chaîne nationale des régions: entre patrimonialisation et «vivre ensemble»

La «politique de fiction» de France 3 est partie prenante de sa stratégie de programmation, basée sur les contraintes de son cahier des charges et de son contrat d'objectifs et de moyens. Ainsi, le cahier des charges 2009 du groupe France Télévisions, qui depuis la réforme de cette même année est devenu société éditrice de programmes, stipule dans son article 11 (décret no 2009-796 du 23 juin 2009) : "L'effort doit porter notamment sur l'adaptation du patrimoine littéraire français et sur l'écriture de fictions abordant et éclairant les pro- 
blématiques et les évolutions de la société contemporaine. La fiction doit également refléter toute la diversité de la société française. » L'article 37 complète ces missions en insistant sur « la lutte contre les discriminations et la représentation de la diversité à l'antenne ", concluant que les chaînes du groupe doivent promouvoir «les valeurs d'une culture et d'un civisme partagés.»

La politique de fiction de France 3 apparaît ainsi insérée dans un cadre régulé et contrôlé a posteriori, d'où une insistance de la chaîne à justifier ses choix de fictions dans le cadre de ses rapports d'activité. Ainsi en 2004:

La chaîne a poursuivi sa politique de fictions portant sur la lutte contre la discrimination, à travers notamment son nouveau feuilleton Plus belle la vie, plusieurs des héros récurrents de la chaîne (Fabien Cosma, SOS 18, Famille d'accueil, Le Camarguais) ou les fictions unitaires : Le pays des enfants perdus, SOS 18, [...], Le Camarguais, Louis la Brocante.

Fiction unitaire en 2004, Louis la Brocante deviendra une série devant le succès du téléfilm. Il est d'ailleurs intéressant de constater que cette série, bénéficiant des meilleures audiences de la chaîne, n'est pas celle qui est le plus mise en avant par l'encadrement de France 3 dans les documents officiels, qui table davantage sur son action en matière de diversité sociale (le rapport 2007 évoquant ainsi la constitution d'un "groupe diversité-fiction »). La série Famille d'accueil est ainsi jugée plus digne, car permettant «d'aborder des thématiques liées à la difficulté des parcours d'immigration et d'intégration » (Rapport 2008).

La politique de fiction de France 3 apparaît ainsi comme une synthèse de ses obligations de service public, joignant des thématiques liées aux questions sociales, à l'immigration, etc., à un cadrage particulier des régions et provinces françaises présentées dans un espace national commun (Lafon, 2000). En effet, on ne peut bien comprendre le positionnement des fictions diffusées sur France 3 que si l'on pose que le modèle français de télévision régionale est la résultante d'un héritage pour une large part centralisateur (celui de la régionalisation de la RTF) et d'une incapacité à réformer cette institution, en dépit des dispositions de la loi de 1982. Ce modèle de télévision nationale à fenêtres régionales s'est ainsi consolidé, fondé sur la proximité comme rhétorique spécifique. Le cahier des charges de France Télévisions de 2009 précise ainsi dans son article 3 :

France 3 : chaîne nationale à vocation régionale et locale, chaîne de la proximité, du lien social et du débat citoyen. La programmation de France 3 contribue à la connaissance et au rayonnement des territoires et, le cas échéant, à l'expression des langues régionales. Dans un monde globalisé, elle offre à chacun la possibilité de réfléchir sur ses racines tout en suivant l'évolution de la société contemporaine en ouvrant une fenêtre sur le monde.Par sa programmation fictionnelle, comment France 3 cherche-t-elle à construire ce « rayonnement des territoires » tout en «ouvrant une fenêtre sur le monde»? 
Afin de comprendre comment la fiction peut contribuer à remplir une telle mission de service public, on peut tenter de partir - comme l'a préconisé Sabine Chalvon-Demersay dans son analyse consacrée aux adaptations télévisées - d'une démarche fondée sur une « sociologie pragmatique des personnages » et sur une «sociologie des processus de création » (Chalvon-Demersay, 2005, p. 79-80). Si les processus de création eux-mêmes ne sont qu'esquissés ici (voir point suivant), ils constituent un point central nous permettant de comprendre la logique générationnelle de production des séries, destinées à des publics de générations spécifiques, et initiées et portées par des sociétés de production ayant une histoire et une culture d'entreprise propres. Nous chercherons ainsi à nous focaliser davantage sur une sociologie pragmatique des personnages qui, ressemblant «à des êtres sociaux tels que les sociologues aimeraient qu'ils soient » (ibid., p. 82), peuvent nous renseigner utilement, par leurs personnalités et leurs actions narrées, sur des orientations morales et normatives communes aux professionnels des médias et aux téléspectateurs ${ }^{1}$.

Ce faisant, par sa consommation médiatique de fictions, chaque téléspectateur participe au quotidien à une expérience collective : «La fiction a, fondamentalement, un effet de désindividualisation : ce en quoi, d'ailleurs, elle concerne très directement les sciences sociales, en particulier la sociologie et l'anthropologie» (Heinich, 2005, p.70). Les séries télévisées renforcent encore davantage ce phénomène par la ritualisation des pratiques de réception imposée par la multiplicité des épisodes : «Les séries sont [...] d'excellents candidats pour jouer dans ce cadre familial et culturel un rôle important» (Esquenazi, 2010, p. 19).

\section{Trois séries dominantes, trois formules distinctes}

Eu égard aux points évoqués précédemment, les fictions diffusées sur France 3 s'insèrent dans un contexte culturel et social bien défini, traversé par les attentes particulières des publics. Les succès d'audience de France 3 laissent apparaître une certaine stabilité (séries, films populaires, magazines éprouvés tels que Thalassa). Selon notre décompte, les séries représentent 43,3\% des meilleures audiences en prime time sur 2005-2011. Il s'agit donc d'un genre majeur pour la chaîne, incarné par les trois séries principales de notre corpus. Le tableau suivant détaille les dix épisodes retenus.

1. Cette démarche, issue d'une longue enquête auprès de producteurs et scénaristes, vise à « partir de ce qui caractérise la fiction télévisée en propre : la largeur de son adresse, sa dimension institutionnelle centralisée, la clarté de son affichage normatif» (Chalvon-Demersay, 2005, p. 80). Elle se réfère ainsi à l'anthropologie de manière renouvelée, pour analyser les processus de production et le contenu des œuvres elles-mêmes (et non d'éventuels « mythes»). 


\begin{tabular}{|c|c|c|c|c|}
\hline Année & Titre de la série & Titre de l'épisode & Rang (1) & $\begin{array}{l}\text { Téléspecta- } \\
\text { teurs (2) }\end{array}$ \\
\hline \multirow{2}{*}{2005} & Louis la Brocante & Louis et la chorale & 2 & 8,145 \\
\hline & Famille d'accueil & Soupçons & 6 & 6,495 \\
\hline $2 \square 06$ & Louis la Brocante & Louis et le mystère du viager & 1 & 9,137 \\
\hline $2 \square \square 7$ & & Louis et le chapitre manquant & 4 & 5,838 \\
\hline $2 \square 08$ & & Louis et le condamné à domicile & 3 & $5,67 \square$ \\
\hline \multirow{2}{*}{$2 \square \square 9$} & Un village français & Le débarquement & 4 & $5,5 \square$ \\
\hline & & Chaos & 3 & 5,617 \\
\hline $2 \square 1 \square$ & Louis la Brocante & Louis et le palais idéal & 2 & $5, \square 8$ \\
\hline \multirow{2}{*}{$\begin{array}{l}2 \square 11 \\
\text { (janv.- } \\
\text { mars) }\end{array}$} & Famille d'accueil & Sortir de l'ombre & 3 & 5,00 \\
\hline & Louis la Brocante & Louis et le monte-en-l'air & 4 & $5,5 \square 1$ \\
\hline
\end{tabular}

(1) Audiences de l'année.

(2) En millions. Source des audiences : Médiamétrie

Tableau 1. Liste et audience des épisodes retenus

Notre objectif dans la constitution de ce corpus est avant tout qualitatif : il s'agit de mettre en évidence les structures dominantes de la programmation sérielle de France 3, afin de caractériser «la structure globale du texte », le texte étant en l'occurrence l'offre sérielle de cette chaîne (Coulomb-Gully, 2002, p. 109). En dépit de leur caractère préconstruit, les indicateurs d'audience nous ont semblé constituer un bon indice de la structure du discours fictionnel proposé par France 3, puisqu'il s'agit de l'expression chiffrée d'une réalité tangible : le choix de téléspectateurs de se livrer à une activité de réception, contrairement à d'autres soirées où ils ont fait un autre choix (autre chaîne ou autre activité), produisant ce qu'Éric Macé nomme un «conformisme instable» ou «provisoire » de la programmation télévisuelle (Macé, 2001, p. 27). Ce conformisme provisoire traduit par conséquent le moment où une offre télévisuelle rencontre un public, rencontre fondée sur le choix d'un «genre » et d'une «formule» (Esquenazi, 2010, p. 89 et suiv.) par ledit public.

Le genre sériel présente ainsi une stabilité remarquable à la télévision (d'où conformisme), les formules quant à elles évoluant progressivement et traduisant des glissements générationnels (d'où provisoire). Un point remarquable est à noter concernant les trois séries qui composent notre corpus : chacune d'entre elles semble se rattacher à un type différent, selon la typologie établie par Jean-Pierre Esquenazi (ibid., p. 102-136). Plus notable encore concernant les sociétés de production à l'origine de ces séries (en collaboration avec France 3) : chacune d'elles semble présenter une marque de fabrique ancrée 
dans son contexte de création, comme si par atavisme les caractères du média télévisuel de ce contexte perduraient dans les séries produites. Ainsi, Louis la Brocante est produite par Télécip (créée en 1969, filiale de Telfrance, créée en 1949), Famille d'accueil par GMT (créée en 1987, filiale du groupe Lagardère) et Un village français par Tetra Media (créée en 1990, société indépendante). Le producteur est en effet «au cœur du dispositif » de par la réglementation française de l'exception culturelle; c'est ce dernier, en liaison avec des auteurs, qui propose aux diffuseurs les programmes (Dagnaud, 2006, p.52). Examinons plus en détail les caractères propres de chacune de ces trois séries.

La plus ancienne, LLB, se rattache davantage au type nodal, chaque épisode narrant une aventure du brocanteur. C'est d'ailleurs la série présentant le caractère le plus traditionnel, avec une référence appuyée à des personnages folkloriques (gendarmes, religieuses). Si «les séries immobiles nodales apparaissent aujourd'hui un peu démodées ", elles peuvent être «encore efficaces» (Esquenazi, 2010, p.106) : concurrencée par le développement des chaînes de la TNT, France 3 continue avec LLB à réaliser de bonnes audiences. On peut d'ailleurs noter que ce caractère très classique est une véritable signature, la facture revendiquée de la convocation de tout un univers bien français : celui des années 1950-1960. Les véhicules des héros, le TUB Citroën de Louis et la 2 CV de la religieuse présents dans chaque épisode, illustrent directement la référence à cette époque. Un autre élément mérite d'être mentionné : celui des intitulés de chaque épisode, construits à la manière des récits pour enfants de cette époque, par exemple et parmi les plus évocateurs : Louis et le secret de l'abbé Cyprien, Louis et le violon noir, Louis et les deux mousquetaires... On retrouve dans ces titres tout l'univers et les promesses de la littérature d'aventure pour la jeunesse ou des livres pour enfants (de Tintin à Martine). C'est donc précisément le caractère immuable de la série qui en assure le succès, opérant la promesse de retrouver et de réactiver les images d'une France intemporelle.

FA propose pour sa part un caractère davantage évolutif, principalement en raison du fait qu'il s'agit d'une famille dont les enfants grandissent. Cependant, cette série reste tout à fait classique, chaque épisode proposant de suivre un enfant ou un adolescent en difficulté placé au sein de la famille. On pourrait donc dire qu'il s'agit d'une série nodale («noyau immobile», Esquenazi, 2010, p.134-135) à caractère évolutif, cette évolutivité tenant à l'actualité des questions sociétales abordées, en lien avec l'évolution des protagonistes dans les âges de la vie. Cette marque de fabrique se retrouve dans l'autre série à succès produite par GMT, Julie Lescaut, qui, comme FA, est une «série à héros édifiants» de cette société de production (Dagnaud, 2006, p. 107).

Enfin, VF se rapproche davantage des genres évolutifs, et plus précisément du type choral. Il s'agit bien de proposer la «biographie d'une communauté» (Esquenazi, 2010, p.119) tout en conservant un aspect feuilletonesque. La communauté concernée par la narration est un village français sous l'Occupation, les 
membres de la communauté interagissant et développant des stratégies face à l'adversité comme le résume le scénariste Frédéric Krivine : "C'est une comédie humaine passée au shaker de l'Occupation » (Libération, 30 mai 2009). Là encore, la production joue un rôle majeur. Cette série s'insère dans un contexte marqué depuis les années 1990 par le renouveau des séries américaines et leurs succès en première partie de soirée. Le format de 52 minutes est ainsi adopté dès son lancement par Tetra media, contrairement aux séries de GMT évoquée précédemment, qui se plieront à cette nouvelle durée afin de renouveler leur offre. Cette prise en compte d'un format proprement télévisuel et non issu de la diffusion en salles va de pair avec l'émergence d'une nouvelle génération de scénaristes qui «cherchent à séduire le plus grand nombre » et « trouvent stimulant de jouer avec les contraintes et les filtres normatifs» (Dagnaud, 2006, p. 88).

Il apparaît, au terme de cette rapide qualification des trois séries présentes dans le corpus, une certaine évolution du genre, intégrant les nouvelles formes sérielles plus évolutives et un format plus court (52 minutes), privilégié par FA et VF. La correspondance entre la formule de ces séries et la nature de leur société de production en est certainement une explication, reposant sur une variable générationnelle. Il convient de noter toutefois que ces trois séries coexistent dans la grille des programmes de France 3 et présentent certains traits communs liés à leur ancrage régional. Ce faisant, en dépit de leurs spécificités, proposent-elles un « espace identitaire collectif» (Dagnaud, 2006, 244) commun? Dans une optique d'analyse sociologique pragmatique des personnages, qui sont ces individus fictionnels français et provinciaux mis en scène sur France 3 , et à quelles situations sont-ils confrontés afin d'y apporter une réponse jugée «recevable» (Chalvon-Demersay, 2005, p. 82) par les publics?

\section{Variations sur les provinces françaises, les citoyens et les injustices : des « itérations » en évolution?}

Sélectionnées par un même diffuseur, France 3, les trois séries de notre corpus présentent un fonds commun. Le cadre y est majoritairement rural, les personnages qui s'y meuvent incarnent des rôles sociaux spécifiquement français: «Prosaïquement, l'organisation économique du secteur audiovisuel pousse à l'émergence de programmes porteurs d'un imaginaire national» (Dagnaud, 2006, p. 244). Phénomène renforcé par l'itération, mécanisme permettant aux producteurs de télévision de minimiser leurs risques:

Ce média n'est pas seulement fondé sur la sérialisation, qui implique la répétition d'un dispositif, d'un style et l'apparition d'un personnage familier, mais fonctionne aussi à l'itération : la permanence d'effets et de procédés qui reviennent dans chaque genre d'émission et peuvent même être transversaux. (Ibid., p.149-150).

On verra comment les itérations relatives à la France des provinces sont un 
moyen efficace de poser une intrigue dans un cadre normé et balisé pour les téléspectateurs. Cela étant, ces séries laissent apparaître des variations sensibles, eu égard à leurs formules distinctes et évolutives. Afin de prendre la mesure de ces évolutions (qui côtoient cependant la permanence de formules plus anciennes et éprouvées), notre analyse se déroule en trois temps :

- une approche de la «mise en phase» des téléspectateurs par l'analyse des plans d'ouverture et de «l'effet générique» (Odin, 2000, p. 76);

- une analyse des personnages - «organisateurs de la structure narrative» et «lieux d'investissement pour le lecteur» (Lits, 2008, p. 137 et suiv.);

- dans la lignée de l'analyse des «scènes capitales » et du « dénouement » menée par Sabrina Chalvon-Demersay (2005, p. 88), nous rechercherons enfin le leitmotiv de chaque épisode, en prenant appui sur l'analyse thématique structurale proposée par Roger Mucchielli et dérivée du modèle actantiel de Greimas (Mucchielli, 2006, p. 131).

\section{La «mise en phase : des séquences d'ouvertures porteuses d'un espace identitaire collectif}

La fiction, par ses ressorts émotionnels, propose à ses publics une immersion, rendue possible par la mise en phase : «Par mise en phase, j'entends le processus qui me conduit à vibrer au rythme de ce que le film me donne à voir et à entendre. [...] Familièrement, on désigne ce processus en disant que l'on a "marché” »(Odin, 2000 : 38-39). Ainsi, l'analyse des séquences d'ouverture (saynète introductive lorsqu'elle est présente, générique, scène d'ouverture) des dix épisodes composant le corpus fait apparaître un certain nombre de schémas récurrents, au premier plan desquels la mise en images de la campagne française. Les paysages sont largement filmés dans les premiers instants de la plupart des épisodes considérés : plus précisément, il s’agit du cheminement sur une route de campagne, qu'il s'agisse du TUB Citroën (fourgon français mythique, autrement appelé HY) de Louis la Brocante, de la voiture d'un éducateur amenant une petite fille dans Famille d'accueil ou de villageois cherchant à rentrer chez eux dans Un village français. La quasi-totalité des lieux mis en image dans les séquences d'ouverture et dans les génériques sont par conséquent des lieux de la ruralité française, filmés avec soin dans l'optique de la mise en phase évoquée précédemment. Cependant, par-delà cette ruralité omniprésente, les séries étudiées laissent entrevoir de nettes différences, propres à la formule de chacune.

Série traitant des questions sociétales contemporaines (adolescence, violences, immigration...), FA propose des ouvertures fondées sur la rencontre avec un enfant ou un jeune, bientôt accueilli dans la famille Ferrière. Le générique joue de ce fait un rôle majeur : l'arrière-plan, essentiel, figure la maison familiale en clôture du générique. Cette maison de famille bourgeoise est celle de l'accueil des jeunes «à problèmes», le lieu de leur réinsertion (réussite politique). 


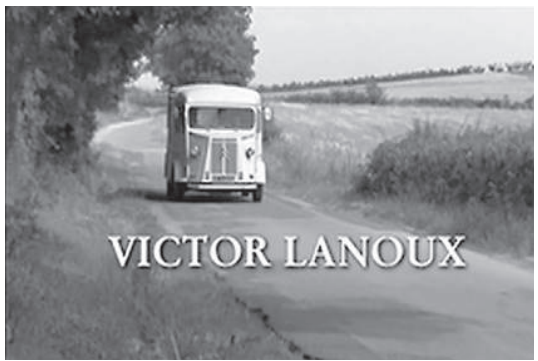

(C) Télécip - France 3

LLB. Louis et le Palais idéal (2010), générique

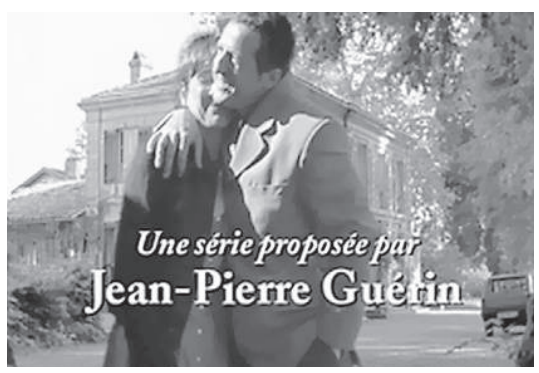

(c) GMT Productions

(avec l'aimable autorisation

de France Télévisions)

FA. Soupçons (2005),

clôture du générique de début

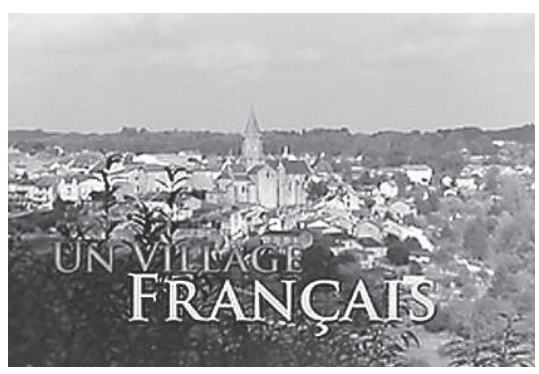

(c) Tetra media / Terego

VF. Le débarquement et Chaos (2009), extrait du générique de début

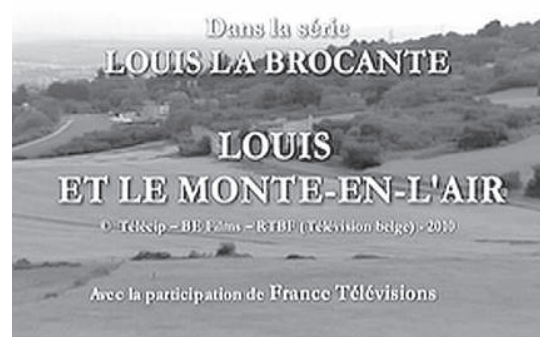

(C) Télécip - France 3

LLB. Louis et le monte-en-l'air (2011), générique

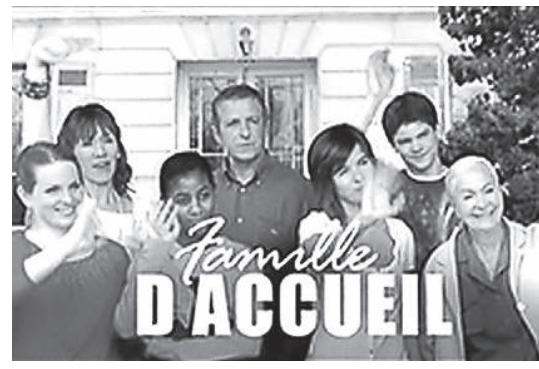

(c) GMT Productions

(avec l'aimable autorisation de France Télévisions)

FA. Sortir de l'ombre (2011), clôt. du gén. de début

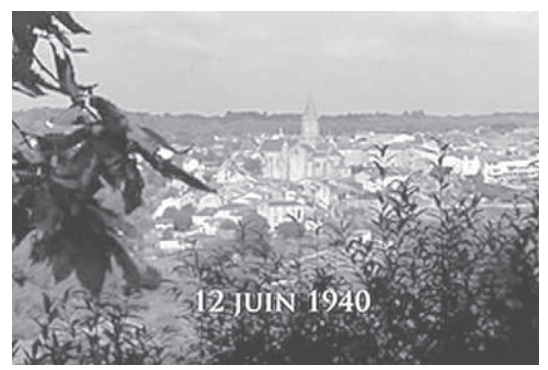

(C) Tetra media / Terego

VF. Le débarquement (2009), plan d'ouverture

Figure 1. Extraits de génériques et de plans d'ouverture d'épisodes du corpus 


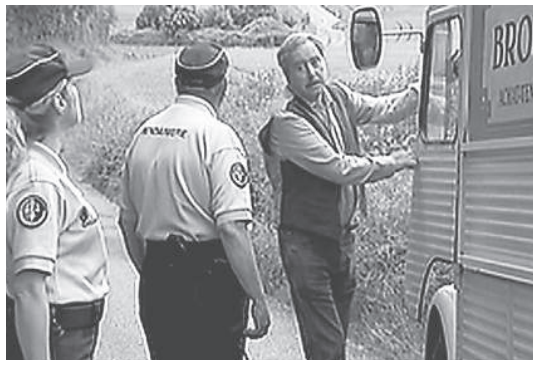

(C) Télécip - France 3

Louis Roman et les deux gendarmes présents à chaque épisode,

Moivin et Raymond.

LLB. Louis et le monte-en-l'air (2011)

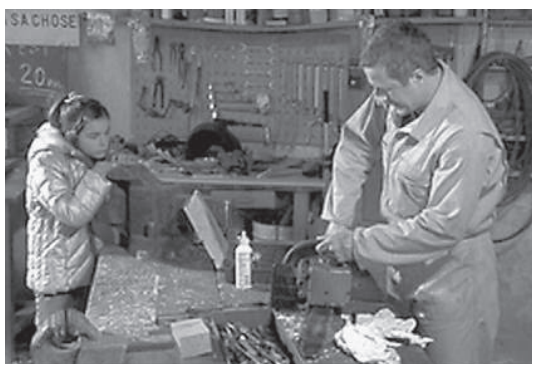

(C) GMT Productions

(avec l'aimable autorisation

de France Télévisions)

Daniel Ferrière, le père, artisan.

FA. Soupçons (2005)

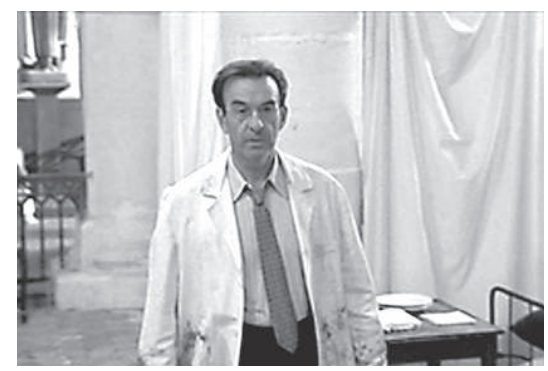

(C) Tetra media / Terego

Daniel Larcher, médecin devenu maire malgré lui VF. Chaos (2009)

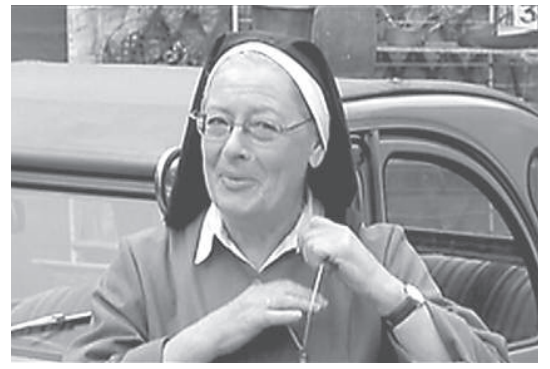

(C) Télécip - France 3

La mère supérieure, responsable de l'orphelinat.

LLB. Louis et le condamné à domicile (2008)

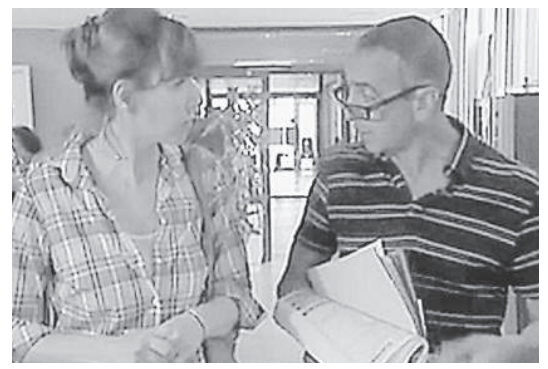

(c) GMT Productions

(avec l'aimable autorisation de France Télévisions) Marion Ferrière, la mère, avec Khaled, directeur de la DDASS, présent à chaque épisode. FA. Sortir de l'ombre (2011)

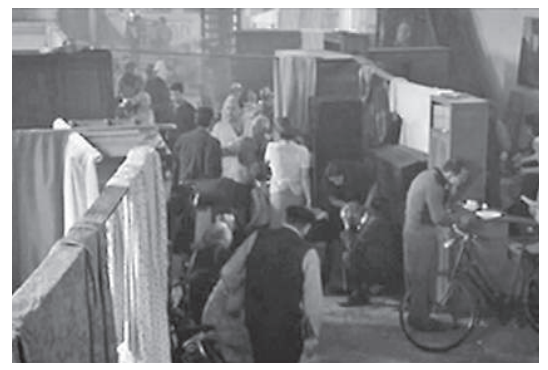

(C) Tetra media / Terego Les habitants du village réunis en quasi-huis clos dans l'église. VF. Chaos (2009)

Figure 2. Personnages fictionnels des épisodes du corpus 
La formule de LLB présente pour sa part une grande stabilité depuis sa création à la fin des années 1990. En l'absence de générique proprement dit (graphiquement parlant), les séquences d'ouverture construites en fonction de chaque épisode se déroulent en deux temps : 1) une série de plans d'ensemble sur la campagne verdoyante (voir illustrations ci-dessus) ; 2) un long travelling qui amène le téléspectateur à suivre le TUB Citroën du héros, qu'il finit par retrouver à la descente du véhicule. Ce procédé itératif de vues esthétisantes des paysages ruraux français est largement utilisé dans d'autres programmes très populaires diffusés par France 3 : épreuves sportives (Tour de France) ou jeu télévisé (La carte au trésor).

Dans un format résolument différent (50 minutes), la série Un village français, lancée en 2009, met au premier plan l'effet générique, ce dernier présentant un caractère extrêmement construit, une «virtuosité stylistique » (Esquenazi, 2010, p. 138) dont on perçoit qu'elle doit beaucoup à la récente influence générique des séries (au sens de genre) : ce ne sont pas moins d'une vingtaine de plans variés qui se succèdent, le tout unifié par une couleur sépia et une musique dramatiquement rythmée. Dès l'ouverture du premier épisode, le générique joue un rôle central et crée une identité visuelle très forte autour des personnages, que l'on retrouvera dans tous les produits dérivés : DVD, site web proposant une immersion vidéoludique dans le village.

Cette rhétorique de la ruralité, d'une France immuable (paysages verdoyants, solide maison bourgeoise, village dominé par un clocher) fondée sur le «souci d'offrir des navigations dans des univers familiers et connotés de la société française » (Dagnaud, 2006, p. 247), est ainsi fortement sollicitée et réitérée dans les séquences d'ouvertures, en dépit de l'évolution des formules. Cette permanence de procédés narratifs, construits par l'interaction auteur-producteur-diffuseur, construit un espace identitaire collectif national : "Les fictions télévisées de chaque pays européen portent une marque de fabrique et versent, avec les émissions d'information et de débats, une multitude d'images dans le fleuve des repères culturels qui façonnent une société » (Dagnaud, 2006, p. 246). Ces repères culturels, ces marques de fabrique nationales, peuvent être aussi mis en évidence par les personnages fictionnels, dont on va tâcher de présenter les caractères propres.

\section{Des personnages choisis, incarnations identitaires françaises}

Les personnages, incarnations du récit, permettent d'établir un lien affectif avec le public et fonctionnent comme un «lieu d'investissement pulsionnel» (Lits, 2008, p.141). Leur analyse peut s'avérer pertinente comme l'a montré Sabine Chalvon-Demersay lorsqu'elle choisit de les considérer «comme de véritables acteurs sociaux » afin "de se donner comme objectif, par une enquête systématique, de retracer la façon dont les personnages, en situation, évaluent 
les situations dans lesquelles ils ont été placés» (2005, p. 81). Ces situations seront abordées dans la partie suivante, une fois que nous aurons mieux caractérisé les personnages des séries du corpus. On peut remarquer de prime abord qu'il s'agit de héros proches des classes moyennes, ne disposant d'aucun capital particulier. Cette spécificité quotidienne des héros de séries télévisées françaises est à rapprocher de l'évolution générale des séries télévisées, gagnées «par un processus croissant de banalisation en se vidant de gestes héroïques au profit des vécus expérientiels et des péripéties qui peuplent le quotidien » (Soulages, 2007, p.111). La figure 2 présente les principaux personnages des épisodes du corpus.

Au vu de la domination de LLB en termes d'audience, les héros fictionnels centraux des soirées de France 3 sont principalement ceux de cette série. Cette dernière est systématiquement construite autour de trois groupes de personnages : le héros - brocanteur de la région lyonnaise - et son entourage familial; les gendarmes; la mère supérieure (et d'autres religieuses). Le héros, en premier lieu, bénéficie d'un patronyme, Louis Roman, connoté de toute la tradition littéraire populaire évoquée plus haut. Ce choix ne doit rien au hasard, "le choix du nom [étant] déterminant dans la caractérisation du personnage, qu'il soit anodin ou de type parodique» (Lits, 2008, p.138). Destiné à vivre des aventures et des mystères à éclaircir, Louis Roman, moustachu bourru au grand cœur incarné par le populaire comédien Victor Lanoux se voit adjoindre un alter ego non moins bourru: la mère supérieure, religieuse catholique dynamique roulant en 2 CV Citroën. Ce type de figuration humoristique de la religieuse s'ancre là encore dans toute une généalogie de personnages fictionnels (série de films sur les gendarmes de Saint-Tropez, série sœur Thérèse.com de TF1, personnages de BD...). Enfin, pour clore ce tableau d'une France intemporelle, la figure du gendarme est systématiquement convoquée, via Moivin et Raymond, duo borné cherchant des noises à Louis. Là encore, la tradition fictionnelle est riche et a déjà fait l'objet d'analyses spécifiques : «Les éléments du paysage gendarmique convoquent un langage récurrent : le décor bucolique d'une France des champs et des clochers constitue le cadre de la mise en scène de la condition gendarmique» (Galera, 2003, p. 116-117). Le gendarme est donc l'incarnation de cette France rurale et tranquille, les usages de son langage sont populaires et «contrastent avec ceux de la société parisienne» (Ibid., p. 120).

Les autres personnages centraux des séries de notre corpus complètent sans la dépareiller cette galerie de héros récurrents à caractère provincial. Ainsi dans la série Famille d'accueil : Tante Jeanne (âgée et un peu bougonne), Daniel Ferrière (père de famille artisan dont l'atelier sert de refuge à des enfants en difficulté), Khaled (responsable de la DDASS)... Ces personnages ancrent les récits dans un réel plus actuel et permettent d'aborder les problématiques sociétales dans leurs aspects de travail social, faisant œuvre de pédagogie «citoyenne».

Pour terminer cette approche, soulignons enfin que les personnages de la 
série Un village français permettent d'identifier des rôles sociaux traditionnels afin d'explorer la réaction de ceux-ci dans leur confrontation avec une situation de chaos : le médecin (Daniel Larcher), notable devenu maire, le patron d'une scierie incarnant une certaine bourgeoisie, son frère communiste, ou encore le policier aigri et fataliste aux prises avec un jeune inspecteur des RG. Il s'agit d'un récit construisant toute une communauté réduite à quelques figures (modèle «choral»), qui incarnent des choix politiques personnels à un moment crucial de l'histoire de France. Ce faisant, ces personnages présentent une ambivalence recherchée, construite par les producteurs. Une projection publique relatée par la presse provoque ainsi des réactions contrastées du public : «Virginie, 27 ans, [...] juge aussi la série crédible : "Par exemple, mon père ne garde pas un mauvais souvenir des Allemands. Ils ont même aidé sa grand-mère à trouver de la pénicilline quand il était malade." Un avis que ne partage pas Cathy, 50 ans. » (Le Parisien, 20 octobre 2009) Le débat suscité par la fiction semble ainsi alimenter les démarches historiennes : cette fictionnalisation d'un quotidien historicisé, défendue par l'historien Jean-Pierre Azéma, conseiller historique de la série, se trouve critiquée par d'autres historiens, notamment Alain Corbin pour qui d'autres voies que le "tournant émotionnel» sont possibles pour amener les non-spécialistes à s'intéresser à la recherche historique (Corbin, 2011, p.61). Cette nouvelle formule de série cherche ainsi à se frayer une place dans l'espace identitaire français, selon un mécanisme décrit par Sabine Chalvon-Demersay (2005, p.107) en ces termes :

La fiction à la télévision contribue à la diffusion d'un évolutionnisme normatif qui ne joue pas seulement par la mise en place de héros positifs porteurs de valeurs explicites mais qui agit principalement en actionnant les ressorts de la temporalité : elle oppose les anciens modèles (dépassés, poussiéreux, ennuyeux), aux nouveaux modèles qui sont plus brillants, plus attrayants.

\section{Des leitmotiv fondés sur des ressorts d'actions normées: centre vs périphérie, pouvoirs vs citoyens}

Les fictions sérielles diffusées sur France 3, ni vains divertissements ni œuvres artistiques, présentent pour chacune une problématique propre, un ressort d'action impliquant les personnages. Dans la lignée de l'analyse des «scènes capitales » et du « dénouement » (Chalvon-Demersay, 2005, p. 88), nous allons, afin d'identifier ce ressort, adopter une méthode d'analyse issue du courant structuraliste, la recherche du leitmotiv. En effet, le leitmotiv d'un récit est selon Roger Mucchielli son «thème général immanent dont les formulations concrètes pourraient varier presque à l'infini », c'est une forme dynamique donatrice de sens (Mucchielli, 1974, p.131). Afin de procéder à cette analyse, nous avons construit un tableau, indiquant un bref résumé de chaque épisode et isolant leur leitmotiv. 
L'examen de ce tableau montre la régularité des leitmotiv mobilisés par ces fictions. Un certain nombre d'entre eux sont récurrents, comme par exemple les abus de la justice et de la police, la question des secrets de famille et du poids de l'héritage, mais aussi et surtout la fragilité de certains acteurs sociaux (personnes âgées, handicapés, jeunesse, personnes en situation précaire...). Sur la base d'une analyse proposée par Roger Mucchielli, dérivée du modèle actanciel de Greimas, à savoir une analyse thématique structurale (Mucchielli, 1974, p. 132 et suiv.), nous pouvons tenter de situer ces divers leitmotiv sur un schéma synthétique. Cette schématisation permet de synthétiser in fine les principaux éléments des leitmotiv recensés dans les dix épisodes du corpus et d'indiquer leurs articulations. Ce faisant, nous percevrons mieux les ressorts qui font agir les personnages.

Il apparaît, au vu de ce schéma, une opposition fondamentale entre, d'une part, les préjugés sociaux (ainsi que le poids de l'héritage familial), générateurs d'abus qui entraînent un découragement du personnage situé au cœur de l'intrigue, et d'autre part un refus de la fatalité, renforcé par la valorisation de la fragilité du personnage dont le portrait donne lieu au récit. Le rôle des héros évoqués plus haut consiste alors à lutter contre les pouvoirs établis et les préjugés afin de provoquer l'émergence d'une nouvelle situation, plus épanouissante.

En ce sens, chacun des récits fictionnels analysés dans le cadre de cette étude présente un fonds politique en ce qu'il implique une prise de position au sein d'une communauté, souvent à l'encontre de positions de pouvoir établies. Monique Dagnaud (2006, p. 257) a bien montré cette tendance du média télévisuel :

Par le biais de la télévision, cette classe moyenne mythique à laquelle s'identifient curieusement les Français [...] serait mise en scène avec ses désarrois, ses révoltes et ses incertitudes face aux puissants, et les téléspectateurs se fédéreraient autour de cette photographie : un imaginaire national à mille lieux de la complexité et de la diversité de la société française.

La télévision reste bien de ce point de vue un média populaire en quête perpétuelle d'une légitimité auprès de ses publics, auxquels elle propose ces récits où triomphent la justice et l'équité, fondées sur un sens commun partagé.

\begin{tabular}{|c|c|c|}
\hline Épisodes & Résumé & Leitmotiv \\
\hline $\begin{array}{l}\text { Louis la Brocante } \\
\text { Louis et la chorale }\end{array}$ & $\begin{array}{l}\text { L'orphelinat dirigé par la mère supé- } \\
\text { rieure est menacé de fermeture par } \\
\text { l'évêché, sur fond de querelles liées à } \\
\text { la propriété de livres anciens (orphe- } \\
\text { linat ou évêché?) qui disparaissent, } \\
\text { la voleuse étant empêtrée dans ses } \\
\text { secrets de famille. }\end{array}$ & $\begin{array}{l}\text { Fragilité des petites struc- } \\
\text { tures et des individus } \\
\text { lorsqu'ils sont dépassés } \\
\text { par des enjeux (enjeux } \\
\text { de pouvoir, secrets de } \\
\text { famille). }\end{array}$ \\
\hline
\end{tabular}




\begin{tabular}{|c|c|c|}
\hline $\begin{array}{l}\text { Louis et le mystère } \\
\text { du viager }\end{array}$ & $\begin{array}{l}\text { Un vieil excentrique propriétaire } \\
\text { d'un château, abusé par son neveu } \\
\text { et sa jeune compagne, qui lui versent } \\
\text { pourtant une rente viagère. }\end{array}$ & $\begin{array}{l}\text { Fragilité des personnes } \\
\text { souffrant de solitude, de } \\
\text { vieillesse et de faiblesse } \\
\text { psychologique. }\end{array}$ \\
\hline $\begin{array}{l}\text { Louis et le chapitre } \\
\text { manquant }\end{array}$ & $\begin{array}{l}\text { Un héritage divise un frère et sa sœur } \\
\text { qui n’ont pas les mêmes projets pour } \\
\text { la maison familiale. Les origines de la } \\
\text { famille sont en question (légitimité, } \\
\text { illégitimité de la descendance). }\end{array}$ & $\begin{array}{l}\text { Poids de l'héritage fami- } \\
\text { lial, lesté de lourds secrets } \\
\text { de famille : la place de } \\
\text { chacun n'est pas forcé- } \\
\text { ment celle que l'on croit. }\end{array}$ \\
\hline $\begin{array}{l}\text { Louis et le condamné } \\
\text { à domicile }\end{array}$ & $\begin{array}{l}\text { Le neveu de Louis, naïf et immature, } \\
\text { est abusé par des voyous et se trouve } \\
\text { aux prises avec un certain acharne- } \\
\text { ment judiciaire. }\end{array}$ & $\begin{array}{l}\text { Fragilité de la jeunesse } \\
\text { immature confrontée à la } \\
\text { malhonnêteté et à l'achar- } \\
\text { nement judiciaire. }\end{array}$ \\
\hline Louis et le palais idéal & $\begin{array}{l}\text { Le neveu de Louis se trouve floué par } \\
\text { deux jeunes filles qui utilisent leur } \\
\text { frère autiste (reproductions artis- } \\
\text { tiques), lequel sera aidé par Louis } \\
\text { après que ce dernier a été inquiété } \\
\text { par la gendarmerie. }\end{array}$ & $\begin{array}{l}\text { L'art n'est pas nécessai- } \\
\text { rement un marché, il peut } \\
\text { être une thérapie et doit } \\
\text { rester une liberté : les abus } \\
\text { policiers vengés. }\end{array}$ \\
\hline $\begin{array}{l}\text { Louis et } \\
\text { le monte-en-l'air }\end{array}$ & $\begin{array}{l}\text { Un jeune homme contraint de voler } \\
\text { pour un antiquaire peu scrupuleux, } \\
\text { afin d'empêcher l'expulsion de sa } \\
\text { famille aux prises avec un agent } \\
\text { immobilier malhonnête. }\end{array}$ & $\begin{array}{l}\text { Fragilité des personnes } \\
\text { en situation de précarité } \\
\text { face aux profiteurs qui les } \\
\text { exploitent. }\end{array}$ \\
\hline $\begin{array}{l}\text { Famille d'accueil } \\
\text { Soupçons }\end{array}$ & $\begin{array}{l}\text { Une petite fille est placée dans la } \\
\text { famille Ferrière à la suite d'accusa- } \\
\text { tions d'inceste. Le père et la mère } \\
\text { s'accusent mutuellement, un profes- } \\
\text { seur se trouve accusé à tort de pédo- } \\
\text { philie. }\end{array}$ & $\begin{array}{l}\text { Un système familial } \\
\text { malade produit des souf- } \\
\text { frances, y compris pour des } \\
\text { personnes accusées à tort. }\end{array}$ \\
\hline Sortir de l'ombre & $\begin{array}{l}\text { Les difficultés d'un jeune homme } \\
\text { dans sa réinsertion: un parcours } \\
\text { marqué par des logiques d'exclusion } \\
\text { et des abus policiers. }\end{array}$ & $\begin{array}{l}\text { Les préjugés de la société } \\
\text { et de la police stigmatisent } \\
\text { les jeunes en voie de réin- } \\
\text { sertion. }\end{array}$ \\
\hline $\begin{array}{l}\text { Un village français } \\
\text { Le débarquement }\end{array}$ & $\begin{array}{l}\text { Malgré l'incrédulité des habitants } \\
\text { et leur inconscience (inspecteur } \\
\text { des RG luttant contre les commu- } \\
\text { nistes, notamment chez des réfugiés } \\
\text { espagnols), l'arrivée des Allemands } \\
\text { bouleverse un village tranquille: } \\
\text { barrages, combats et morts, en parti- } \\
\text { culier parmi les enfants de l'école. }\end{array}$ & $\begin{array}{l}\text { Des villageois inconscients } \\
\text { en butte à l'impensable: } \\
\text { l'invasion de l'ennemi et } \\
\text { la mort. }\end{array}$ \\
\hline Chaos & $\begin{array}{l}\text { La lutte du médecin du village devenu } \\
\text { maire malgré lui (négociations avec } \\
\text { les officiers nazis) pour garantir la } \\
\text { sécurité et la santé des habitants } \\
\text { réfugiés dans l'église, en proie à une } \\
\text { épidémie de dysenterie. }\end{array}$ & $\begin{array}{l}\text { Comment réagir face à la } \\
\text { violence de la guerre et à } \\
\text { l'absence de soins et de } \\
\text { secours. }\end{array}$ \\
\hline
\end{tabular}

Tableau 2. Résumé et leitmotiv de chaque épisode du corpus 


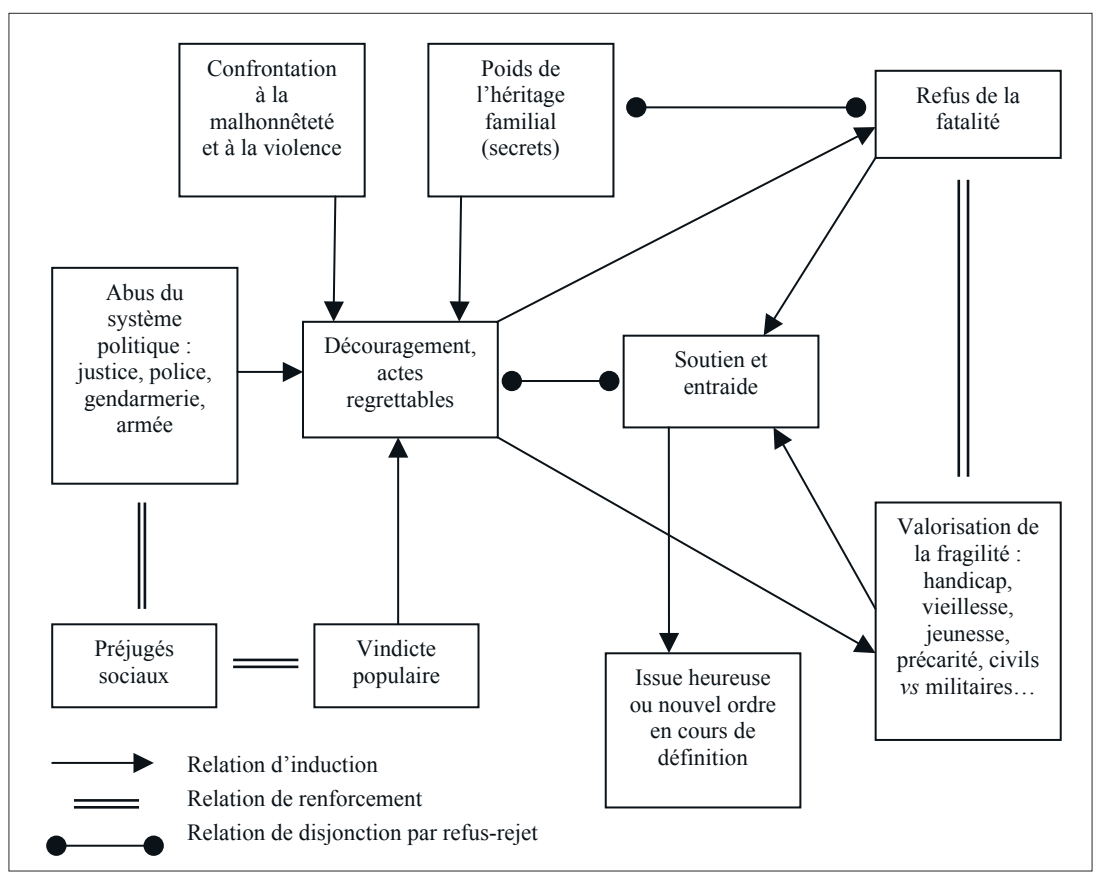

Figure 3. Positionnement des leitmotiv des épisodes du corpus

\section{Références}

CORBIN Alain, 2011, "Les historiens et la fiction. Usages, tentation, nécessité.... ", Le Débat, nº165, p. 57-61.

Chalvon-Demersay Sabine, 2005, «Le deuxième souffle des adaptations ", L'Homme, $n^{0} 175-176$, p. $77-111$.

Coulomb-Gully Marlène, 2002, "Propositions pour une méthode d'analyse du discours télévisuel », Mots. Les langages du politique, n070, p.103-112.

Dagnaud Monique, 2006, Les artisans de l'imaginaire. Comment la télévision fabrique la culture de masse, Paris, Armand Colin.

ESQUENAZI Jean-Pierre, 1998, "Conditions d'un espace public télévisuel régional», colloque «Télévision et espace régional. Politiques, productions, représentations (1949-1997) », Aix en Provence, 25-27 septembre 1997.

- 2010, Les séries télévisées. L'avenir du cinéma?, Paris, Armand Colin.

GoffmAn Erving [1974] 1991, Les cadres de l'expérience, trad. de l'anglais I. Joseph, Paris, Minuit.

GALERA Yann, 2003, "Quand Orphée rencontre Pandore. Le gendarme en ballades», Sociétés \& Représentations, n 16, p. 115-130. 
HEINICH Nathalie, 2005, «Les limites de la fiction », L'Homme, nº 175-176, p.57-76.

LAFON Benoit, 2000, "La télévision régionale, socio-histoire d'un dispositif d'intégration politique nationale. Le cas des journaux télévisés midi-pyrénéens (19632000)", thèse de science politique soutenue le 15 décembre 2000 à l'Université des Sciences sociales, Toulouse 1.

- 2007, «Le cancer en prime time. Émergence d'une confrontation distanciée avec la maladie par des fictions télévisées », Questions de communication, n¹1, p.129-136.

LITS Marc, 2008, Du récit au récit médiatique, Bruxelles, De Boeck.

MACÉ Éric, 2006, Les imaginaires médiatiques. Une sociologie postcritique des médias, Paris, Éditions Amsterdam.

MucCHIELLI Roger, [1974] 2006, L'analyse de contenu des documents et des communications, Issy-Les-Moulineaux, ESF.

ODIN Roger, 2000, De la fiction, Bruxelles, De Boeck.

SCHAEFfer Jean-Marie, 1999, Pourquoi la fiction?, Paris, Le Seuil.

SOULAGES Jean-Claude, 2007, Les rhétoriques télévisuelles. Le formatage du regard, Paris, INA - De Boeck. 